\title{
Advances and perspectives in discovery and functional analysis of small secreted proteins in plants
}

Xiao-Li Hu², Haiwei Lu $\mathbb{0}^{2}$, Md Mahmudul Hassan $\mathbb{D}^{2}$, Jin Zhang ${ }^{3}$, Guoliang Yuan ${ }^{24}$, Paul E. Abraham², Him K. Shrestha ${ }^{2,5}$, Manuel I. Villalobos Solis², Jin-Gui Chen $\mathbb{B}^{2,4}$, Timothy J. Tschaplinski ${ }^{2,4}$, Mitchel J. Doktycz ${ }^{2}$, Gerald A. Tuskan ${ }^{4}$, Zong-Ming (Max) Cheng ${ }^{1,6}$ and Xiaohan Yang ${ }^{1,2,4}$

\begin{abstract}
Small secreted proteins (SSPs) are less than 250 amino acids in length and are actively transported out of cells through conventional protein secretion pathways or unconventional protein secretion pathways. In plants, SSPs have been found to play important roles in various processes, including plant growth and development, plant response to abiotic and biotic stresses, and beneficial plant-microbe interactions. Over the past 10 years, substantial progress has been made in the identification and functional characterization of SSPs in several plant species relevant to agriculture, bioenergy, and horticulture. Yet, there are potentially a lot of SSPs that have not been discovered in plant genomes, which is largely due to limitations of existing computational algorithms. Recent advances in genomics, transcriptomics, and proteomics research, as well as the development of new computational algorithms based on machine learning, provide unprecedented capabilities for genome-wide discovery of novel SSPs in plants. In this review, we summarize known SSPs and their functions in various plant species. Then we provide an update on the computational and experimental approaches that can be used to discover new SSPs. Finally, we discuss strategies for elucidating the biological functions of SSPs in plants.
\end{abstract}

\section{Introduction}

Plant small secreted proteins (SSPs) are less than 250 amino acids (aa) in length and can be actively transported out of plant cells ${ }^{1,2}$. In plants, SSPs have been shown to play important roles in various biological processes such as growth, development, reproduction, resistance to abiotic and biotic stresses, and beneficial plant-microbe interactions $^{3-5}$. In general, 30,000-40,000 proteinencoding genes have been reported in individual plant genomes. ${ }^{6}$. Yet hundreds to thousands of SSPs are potentially overlooked in a single plant genome $\mathrm{e}^{7}$ for two

\footnotetext{
Correspondence: Zong-Ming (Max) Cheng (zcheng@utk.edu) or

Xiaohan Yang (yangx@ornl.gov)

'Department of Plant Sciences, University of Tennessee, Knoxville, TN, USA

2Biosciences Division, Oak Ridge National Laboratory, Oak Ridge, TN, USA

Full list of author information is available at the end of the article

These authors contributed equally: Xiao-Li Hu, Haiwei Lu
}

reasons: (1) the SSP space is occupied by many proteins with a length of less than $100 \mathrm{aa}^{2,8}$ and (2) $50 \%$ of the discovered secreted proteins in plants do not have a known signal peptide ${ }^{9}$, both of which create difficulties in SSP annotation using traditional computational approaches $^{10-12}$.

In recent years, the increasing volume of genomics data and the continuously evolving machine learning algorithms have boosted the effectiveness of computationally predicting SSPs. Meanwhile, advances in functional genomics research have accelerated the experimental validation of predicted SSPs and the elucidation of their functional roles. As a result, SSP-focused research has become an emerging area with great potential for growth, as reflected by the rapidly increasing number of publications on SSPs in various organisms, including animals, microbes, and plants. Here with a focus on plant SSPs, we

\section{(c) The Author(s) 2021}

(c) (i) Open Access This article is licensed under a Creative Commons Attribution 4.0 International License, which permits use, sharing, adaptation, distribution and reproduction cc) in any medium or format, as long as you give appropriate credit to the original author(s) and the source, provide a link to the Creative Commons license, and indicate if changes were made. The images or other third party material in this article are included in the article's Creative Commons license, unless indicated otherwise in a credit line to the material. If material is not included in the article's Creative Commons license and your intended use is not permitted by statutory regulation or exceeds the permitted use, you will need to obtain permission directly from the copyright holder. To view a copy of this license, visit http://creativecommons.org/licenses/by/4.0/. 
first summarize the current understanding of SSP biosynthesis and secretion. We then discuss the structures and functions of representative SSPs that are well characterized in various plant species, including model species, food crops, bioenergy feedstocks, and horticultural plants. We also highlight computational tools, experimental approaches, and their combinations used to identify novel SSPs. Finally, we discuss the strategies that have been or can be used to explore the functions of SSPs.

\section{Biosynthesis and secretion of SSPs in plants Biosynthesis of SSPs}

In plants, SSPs have been found to be produced via multiple alternative pathways, as illustrated in Fig. 1. The majority of the characterized SSPs to date are proteolytic cleavage products synthesized via the removal of an $\mathrm{N}$ terminal signal sequence (NSS; also known as N-terminal signal peptide) and/or a pro-domain from larger protein precursors, which can be either nonfunctional or

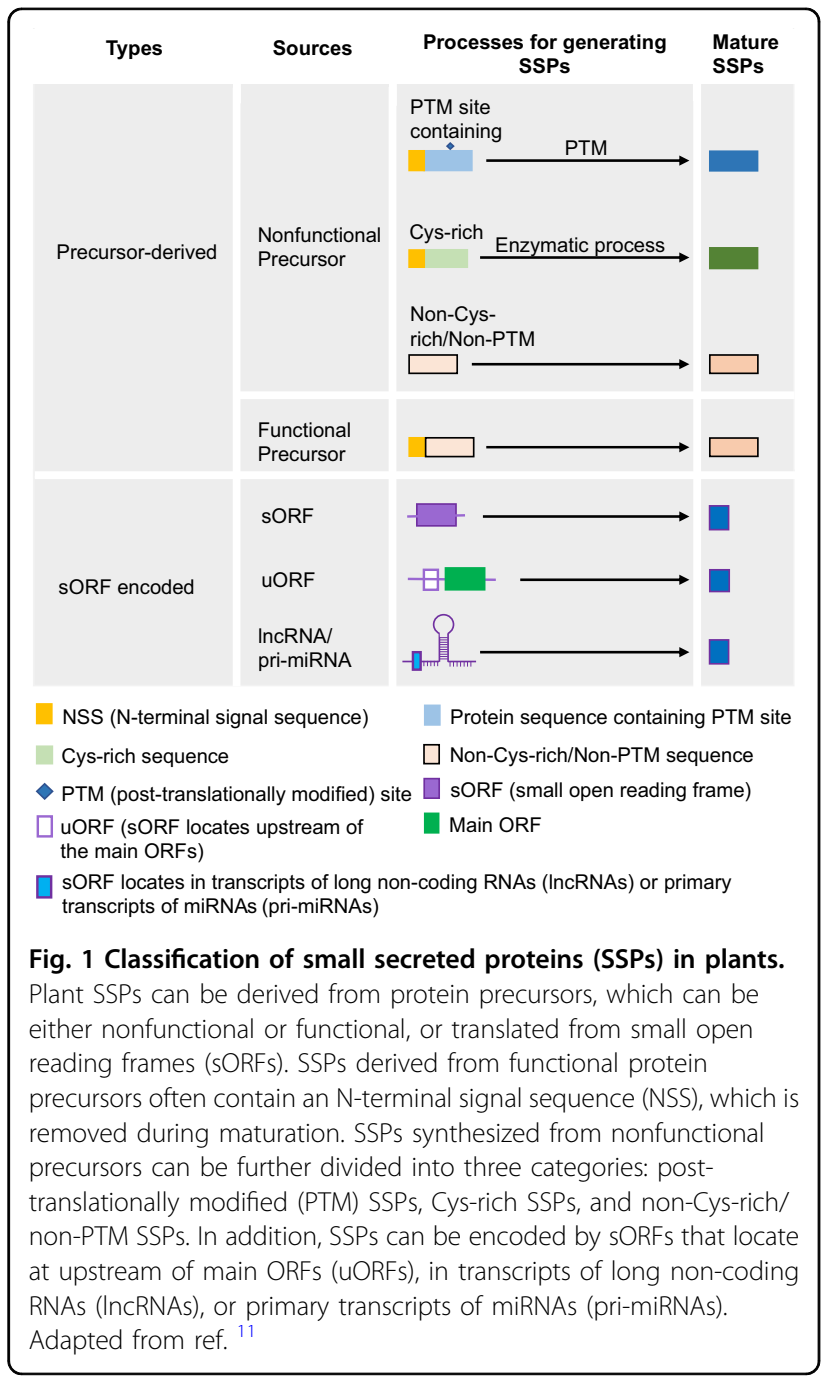

functional ${ }^{11,13}$. SSPs derived from nonfunctional precursors can be further classified into three subcategories based on features of their mature forms. SSPs belonging to the first subcategory typically consist of less than 20 aa in their mature forms which have few or no cysteine (Cys) residues and contain one to several types of posttranslational modifications (PTM), such as tyrosine (Tyr) sulfation, proline (Pro) hydroxylation or Pro glycosylation. Therefore, these SSPs are named PTM SSPs. Several well-studied PTM SSPs in Arabidopsis thaliana are involved in plant growth and development, including CLAVATA 3 (CLV3), C-TERMINALLY ENCODED PEPTIDE 1 (CEP1), PLANT PEPTIDE CONTAINING SULFATED TYROSINE 1 (PSY1), and ROOT MERISTEM GROWTH FACTOR 1 (RGF1) $)^{11,14,15}$. The second subcategory features SSPs with mature peptides that contain an even number (often ranging from 2 to 16) of Cys residues. These Cys residues are essential for forming the disulfide bonds in the active mature SSPs. Most of the known Cys-rich SSPs are involved in plant-microbe interactions, such as PLANT DEFENSINs (PDFs), nonspecific LIPID TRANSFER PROTEINS (nsLTPs), and KNOTTINs. Meanwhile, several Cys-rich SSPs have been found to regulate plant development, such as S-LOCUS CYSTEINE-RICH PROTEIN/S-LOCUS PROTEIN11 (SCR/SP11) and LUREs ${ }^{11,15}$. The third subcategory contains non-Cys-rich/non-PTM SSPs, which often lack the NSS in their precursor forms and contain Cys, Pro, Tyr, glycine (Gly), lysine (Lys), or other amino acids with dominant roles in conferring the activity of the mature SSPs. SSPs within this subcategory have been primarily found to participate in plant defense responses, with SYSTEMINS (SYS), GRIM REAPER PEPTIDE (GRIp), and PLANT ELICITOR PEPTIDES (PEPs) being the representative examples ${ }^{11}$.

In the past decade, a growing number of plant SSPs has been found derived from functional protein precursors, such as INCEPTINs from A. thaliana, Zea mays, Oryza sativa, and Vigna unguiculata, the Glycine max SUBTILASE PEPTIDE (Gm-SUBPEP), and the Solanum lycopersicum CYSTEINE-RICH SECRETORY PROTEINS, ANTIGEN5, and PATHOGENESIS-RELATED 1 PROTEINS derived peptide 1 (CAPE1) ${ }^{11}$.

In addition to being processed from larger protein precursors, plant SSPs can be directly encoded by small open reading frames (sORFs), which can sometimes locate upstream of the main ORFs (therefore called "uORFs"), within presumed non-coding RNAs (e.g., long non-coding RNAs), or within primary transcripts of miRNAs. These SSPs are denoted as "short peptides encoded by sORFs", "sPEPs", or "nonprecursor-derived peptides"11,16,17. Some known examples of such SSPs include the uORF2encoded sucrose control peptide (SC-PEPTIDE) that is required for sufficient sucrose-induced repression of 


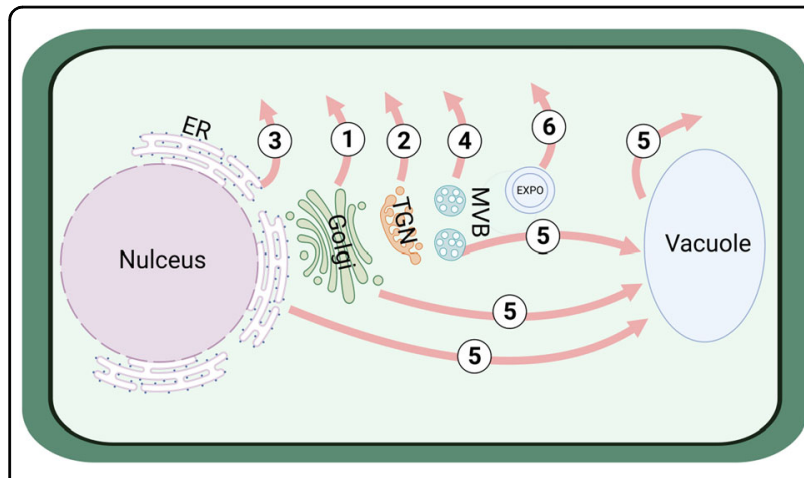

Fig. 2 Secretion mechanisms of small secreted proteins (SSPs) in plants. Most N-terminal signal sequence (NSS)-containing SSPs are secreted via the conventional protein secretion (CPS) pathway that begins at the endoplasmic reticulum (ER). SSPs are subsequently routed through either the Golgi apparatus (Golgi) (1) or trans-Golgi network (TGN) (2) before being delivered to the apoplast.

Alternatively, some NSS-containing SSPs are secreted by unconventional protein secretion (UPS) routes, including direct transportation from ER to the apoplast (3), transportation through secretory multivesicular bodies (MVBs) (4), and vacuoles (5). Cytosolic leaderless proteins (LSPs) are secreted through the excyst-positive organelle (EXPO) (6). Adapted from ref. ${ }^{22}$

translation in $A$. thalian ${ }^{18}$, the miPEP171b that regulates root development in Medicago truncatula ${ }^{19}$, and ENOD40s that are involved in sucrose use in nitrogenfixing nodules in G. $\max ^{20}$.

\section{Mechanisms of SSP secretion}

Our knowledge of plant SSP secretion largely overlaps with our understanding of protein trafficking and secretion, which follows several different mechanisms ${ }^{21-23}$. The majority of plant SSPs with an NSS are secreted via the conventional protein secretion (CPS) pathway (Fig. 2), which is conserved among eukaryotes. Guided by the NSS, SSPs are first transported to the endoplasmic reticulum (ER) where the NSS is removed. These SSPs are then exported to the cis side of the Golgi apparatus (Golgi) and further sorted through the Golgi or the transGolgi network (TGN). Modifications, such as glycosylation that are required for SSP maturation, occur when SSPs travel through the Golgi. Finally, the mature SSPs are delivered to the apoplast via secretory vesicles or granules ${ }^{17,22-24}$.

However, some NSS-containing SSPs bypass the CPS pathway. They follow unconventional protein secretion (UPS) routes (Fig. 2) ${ }^{22,23}$ while traveling to the extracellular space, usually upon pathogen attack or the exposure to other biotic or abiotic stress conditions ${ }^{9,24}$. The simplest UPS route directly transports these proteins from the ER to the plasma membrane (PM). Alternative UPS routes utilize vesicular carriers, including the secretory multivesicular body (MVB) and vacuole, that can fuse with the PM to release their contents into the apoplast/ extracellular space ${ }^{22}$.

In addition, secreted proteins without an NSS (also known as cytosolic leaderless proteins, LSPs), which represent a large proportion of the plant secretome ${ }^{21}$, cannot be processed by the CPS. These proteins have been proposed to be secreted through the excyst-positive organelle (EXPO) - a double-membrane organelle whose formation is Golgi- and TGN-independent. The EXPOs can fuse with the PM to secrete LSPs (Fig. 2) ${ }^{9,21}$.

\section{Known SSPs and their biological roles in plants Known SSPs}

Because the genome of model herbaceous plant $A$. thaliana is considered to be better annotated and characterized than other plant species, we focus on known SSP families found in A. thaliana. Also, we discuss SSPs that have been identified from several important plant species, including Z. mays, O. sativa, $S$. lycopersicum, $M$. truncatula, and Populus trichocarpa. A large number of SSPs have been computationally predicted in plants, as demonstrated in public databases, including OrysPSSP ${ }^{5}$, PlantSSP ${ }^{25}$, and MtSSPdb ${ }^{3}$. For instance, according to the database PlantSSP ${ }^{25}$, there are 2451, 5373, and 3216 predicted SSPs, which are less than 200 aa in length with NSS, in A. thaliana, $O$. sativa, and $P$. trichocarpa, respectively. These predicted SSPs account for $6.9 \%, 8.0 \%$, and $7.1 \%$ of all the annotated proteins (including splice variants) in the $A$. thaliana (version TAIR10), O. sativa (version MSU6.1), and $P$. trichocarpa (JGI v2) genome, respectively. More recently, with the release of the reannotated M. truncatula genome, 4439 genes $(6.3 \%$ of all the annotated genes) were predicted to encode SSPs that are less than 230 aa with NSS but not transmembrane regions ${ }^{3}$. Although interest in decoding genomes for potential SSPs has been growing substantially in recent years, only a limited number of SSPs have been experimentally characterized, which are distributed among approximately 50 gene families ${ }^{13}$, with their representative members listed in Table 1.

\section{Structure of known SSPs}

Protein function is dependent on a well-defined and folded three-dimensional (3D) structure and intrinsically disordered regions (IDRs), which are not likely to form a defined 3D structure ${ }^{26}$. Some of the known SSPs in plants have well-defined 3D structure, as demonstrated in Fig. 3. For instance, hydroxyproline-bound tri-arabinoside-induced conformation was found when post-translationally modified protein CLV3 became biologically active ${ }^{27}$. The $\beta$-turn-like conformation, for example, which is a feature of CEP1, is associated with biological activity ${ }^{28}$. On the other hand, enzymatic maturation processes produce bioactive Cys-rich 
Table 1 A list of representative small secreted proteins that have been experimentally confirmed in plants

\begin{tabular}{|c|c|c|c|c|c|}
\hline Plant species & Protein name & Gene locus & Protein length (aa) & Gene family* (Pfam ID) & Reference \\
\hline Arabidopsis thaliana & CEP1 & AT1G47485 & 91 & & 89 \\
\hline Arabidopsis thaliana & CLV3 & AT2G27250 & 96 & PF11250 & 148 \\
\hline Arabidopsis thaliana & EPLF9 & AT4G12970 & 102 & PF16851 & 149 \\
\hline Arabidopsis thaliana & EPF1 & AT2G20875 & 104 & PF13912 & 150 \\
\hline Arabidopsis thaliana & GLV6 & AT2G03830 & 123 & & 151 \\
\hline Arabidopsis thaliana & LTP1 & AT2G38540 & 118 & PF00234 & 152 \\
\hline Arabidopsis thaliana & PREPIP1 & AT4G28460 & 72 & & 153 \\
\hline Arabidopsis thaliana & PREPIP2 & AT4G37290 & 84 & & 153 \\
\hline Arabidopsis thaliana & PROPEP1 & AT5G64900 & 92 & PF00879 & 154 \\
\hline Arabidopsis thaliana & PROPEP2 & AT5G64890 & 109 & PF00879 & 155 \\
\hline Arabidopsis thaliana & PROPEP3 & AT5G64905 & 96 & PF00879 & 55 \\
\hline Arabidopsis thaliana & PSK1 & AT1G13590 & 87 & PF06404 & 156 \\
\hline Arabidopsis thaliana & RALF1 & AT1G02900 & 120 & PF05498 & 157 \\
\hline Arabidopsis thaliana & RGF1 & AT5G60810 & 116 & & 158 \\
\hline Arabidopsis thaliana & IDA1 & AT3G25655 & 86 & & 159 \\
\hline Medicago truncatula & NCR169 & Medtr7g029760 & 61 & PF07127 & 160 \\
\hline Oryza sativa & DEF7 & LOC_Os02g41904.1 & 80 & PF00304 & 161 \\
\hline Populus trichocarpa & CLE20 & Potri.014G156600 & 74 & & 39 \\
\hline Solanum lycopersicum & CAPE1 & Solyc00g174340 & 159 & PF00188 & 162 \\
\hline Zea mays & PROZIP1 & AC210027.3_FG003 & 137 & & 51 \\
\hline
\end{tabular}

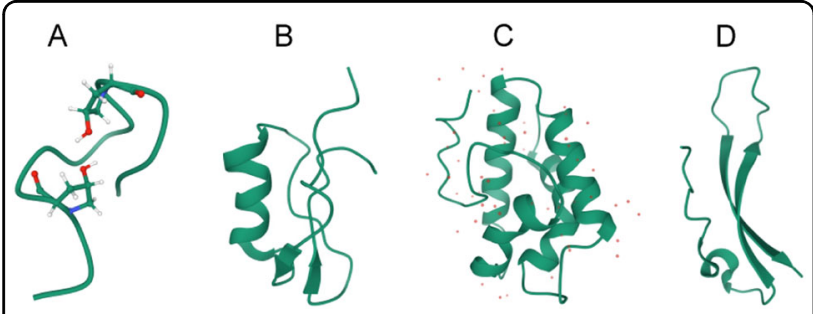

Fig. 3 Three-dimensional structure of some known small secreted proteins in plants. A CEP1 (PDB ID: 2MFO). B SCR/SP11 (PDB ID: 1UGL). C LTP (PDB ID: 1MZL). D Stomagen (PDB ID: 2LIY). The Protein Data Bank (PDB) data were obtained from RCSB protein data bank (https://www.rcsb.org/) ${ }^{165,166}$ and visualized using Mol*167

SSPs with correct oxidative folding under oxidative conditions by

forming diverse disulfide patterns as well as loop regions, which are supposed to be crucial for protein-protein interactions (PPIs) ${ }^{15,29}$. SCR/SP11 contains an $\alpha / \beta$ sandwich motif connected by L1 loop that serve as binding site for specific receptors ${ }^{30}$. LTP has four $\alpha$-helices, three loops, and four disulfide bridges with eight conserved cysteines ${ }^{4}$. EPF includes one loop and three disulfide bonds, which contains two antiparallel $\beta$-strands connected by a 14-residue loop ${ }^{31}$. However, it has been estimated that $10 \%$ of secreted proteins are intrinsically disordered proteins (IDPs), with $>70 \%$ of their length being IDRs ${ }^{26}$. For example, LTP1 from $A$. thaliana contains a defined 3D structural domain (Fig. 3C) and without IDR (Fig. 4A) but LEA4 from A. thaliana has no defined 3D structural domain and is fully disordered (Fig. 4B).

\section{Biological roles of known plant SSPs \\ Role of SSPs in plant growth and development}

Some of the known SSPs are associated with multiple aspects of plant growth and development. During these processes, most SSPs act as signaling molecules that are involved in cell-to-cell communication by binding membrane receptors and coordinating responses with plant hormones ${ }^{14,32}$. In terms of meristem maintenance, CLE14 and CLE40 expression has been observed in A. thaliana root meristematic zone and found to play roles in controlling meristematic activity as well as cell number ${ }^{33,34}$. Although CLE43 does not affect root apical meristem growth in A. thaliana ${ }^{35}$, its homologs, BnCLE43a and 


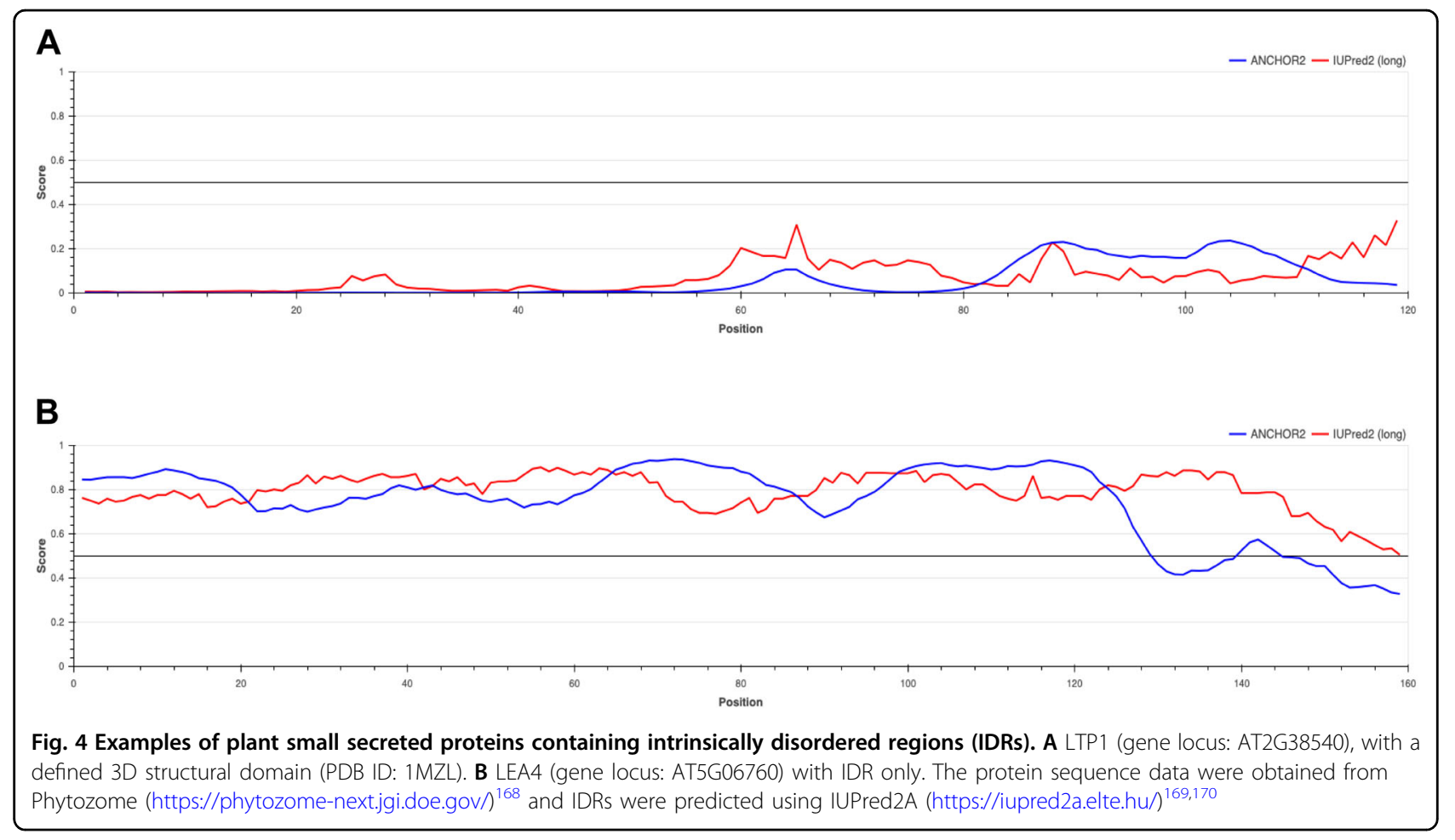

BnCLE43b, were found in Brassica napus could repress $A$. thaliana root growth when synthetic peptides were added to the culture medium ${ }^{36}$. In $A$. thaliana, both CLE9 and CLE10 control xylem differentiation through regulation of the cytokinin signal pathway ${ }^{37}$, and CLE41 can drive vascular cell division ${ }^{38}$. In contrast, PtrCLE20 identified in vascular cambium cells of $P$. trichocarpa was shown to restrain cell division, resulting in an inhibition of lateral growth of the stem $^{39}$. Besides the impact on vegetative tissues or organs, SSPs can affect flower development. For example, CLV1 acts with CLV3 to avoid enlarged meristems and extra floral organs in $A$. thaliana ${ }^{40}$. The pollen-specific SIPRALF gene that encodes a 129 aa preproprotein was recognized to negatively regulate pollen tube elongation in S. lycopersicum ${ }^{41}$.

Role of SSPs in plant response to abiotic and biotic stresses

To sense and respond to various stresses, plants have evolved complex signaling and defense mechanisms ${ }^{42}$. Induced SSPs have been observed in many stress responses in plants, including some SSPs recognized as hormone-like molecules ${ }^{43}$. SSPs act quickly and synergistically at low concentrations in reaction to different stresses $^{44}$.

SSPs are involved in a variety of biotic stresses responses in diverse plant species. For example, an SSP called SYSTEMIN identified in S. lycopersicum was the first wound response signaling peptide ${ }^{45,46}$. When plants are attacked by herbivores or pathogens, a series of defense signals and pathways can be induced by SYSTEMIN through its interaction with SYSTEMIN RECEPTOR 1, which includes stimulation of PROTEASE INHIBITOR production, as well as enhancement of ethylene and jasmonic acid biosynthesis ${ }^{47,48}$.

Plant SSPs can initiate immune responses and increase resistance to pathogens. For example, an SSP called IRP, which was identified from the proteomic analysis of $O$. sativa suspension cells cultured with bacterial peptidoglycan and fungal chitin, increased the abundance of phenylalanine ammonia-lyase 1 (PAL1) and activated mitogen-activated protein kinases (MAPKs), which are known to be associated with plant immunity ${ }^{49}$. Two pathogen-responsive SSPs, TaSSP6 and TaSSP7, are responsible for resistance to Septoria tritici blotch, a severe foliar disease caused by the fungal pathogen Zymoseptoria tritici in Triticum aestivum ${ }^{50}$. In Z. mays, Zip1 was demonstrated to trigger plant immunity by activating salicylic acid defense signaling ${ }^{51}$.

SSPs are also involved in responses to abiotic stresses. For example, CLE25, found in A. thaliana, is induced under dehydration, which triggers ABA biosynthesis in leaves to prevent water loss by regulating stomatal closure $^{52}$. In $A$. thaliana roots, AtRALFL8 encoding a SSP can be induced not only by nematode infection but also by drought stress, leading to cell wall remodeling ${ }^{53}$. To determine extracellular proteins that respond to heat stress, a quantitative proteomic analysis was conducted by collecting proteins from heat-tolerant Sorghum bicolor 
cell suspension culture medium, resulting in the identification of an SSP named germin protein, which was highly induced at the protein level ${ }^{54}$. Another example is the small peptide AtPep3 encoded by AtPROPEP3, which has been shown to play an important role in salinity stress tolerance in A. thaliana ${ }^{55}$.

\section{Role of plant SSPs in beneficial plant-microbe interactions}

SSPs play important roles in cross-kingdom interactions. It is widely accepted that SSPs generated from plant-associated microorganisms (e.g., fungi, bacteria) can be used as effector proteins to promote plant microbial colonization $^{56-58}$. However, studies on the identification of plant SSPs as effector proteins that affect microbes have been very limited ${ }^{2}$. Plants can adapt to a low availability of nutrients by altering root system architecture, with some can form symbiotic associations with rhizobia and mycorrhizal fungi ${ }^{59,60}$. In legumes, SSPs can affect root development and rhizobial-legume symbiosis $^{61,62}$. CLE family members have been characterized in different species, such as CLE12 and CLE13 in M. truncatula, CLERS (CLE-root signal) 1/2/3 in Lotus japonicus, and RIC (rhizobium-induced CLE) in G. max. These SSPs appear to be involved in the negative systemic autoregulation of the nodulation pathway and inhibit newly formed nodules in roots ${ }^{63}$. Conversely, in M. truncatula, CEP1 was found to modulate lateral root formation and increase the number and size of nodules ${ }^{60}$. When $L$. japonicus was inoculated with the arbuscular mycorrhizal (AM) fungus Rhizophagus irregularis, in comparison with formation of nodules in $L$. japonicus, alternate CLE genes, including $L j C L E 19$ and $L j C L E 20$, were upregulated in roots, indicating that different signaling pathways are involved in $\mathrm{AM}$ and root nodule symbiosis ${ }^{64}$. In addition, a recent study reported that SSPs produced by $P$. trichocarpa were induced when co-culture with ectomycorrhizal mycorrhizal (EM) fungus Laccaria bicolor and several P. trichocarpa SSPs could enter fungal hyphae when they were exposed to L. bicolor ${ }^{2}$, suggesting plant SSPs may mediate ectomycorrhizal symbiosis as well.

\section{Computational and experimental approaches for discovery of SSPs in plants \\ Computational approaches for discovery of SSPs}

In general, there are two main steps to computationally predict SSPs in plant genomes, i.e., predicting small proteins encoded by sORFs and subsequently evaluating their ability to be secreted. A large number of sORFs can be found by locating in-frame start and stop codons in the plant genomes. However, annotations of sORFs have been largely overlooked because such short sequences were initially classified as random nonsense occurrences ${ }^{65}$. In the recent decade, progress in the development of computational methods for gene prediction has contributed to the identification of numerous SORFs in plants. For example, sORF finder is a tool for identifying putative small sORFs between 10 and 100 amino acids based on significant selective constraints, which works well for predicting sORFs in plant genomes ${ }^{66}$. Small Peptide Alignment Discovery Application is a homology-based program which can accurately identify and annotate genes in a given family, including sORFs in plants ${ }^{67}$. One caveat of these in silico SORF prediction tools is that the predicted sORFs may be pseudogenes. To address this issue, transcript expression data generated by transcriptome sequencing (RNA-seq) can be used for identifying functional sORFs, as demonstrated in SSP discovery in $P$. trichocarp ${ }^{2,10}$. Transcript sequences obtained from RNA-seq data can be either protein coding sequences (CDS) or non-coding RNAs ${ }^{68,69}$. Finally, using DeepCPP, a new deep neural network-based tool, aims to predict short sequences with coding potential ${ }^{70}$.

The potential for secretion of small proteins has been determined using tools based on specific algorithms, in particular many use newly developed machine learning (ML) approaches (Table 2). To predict NSS-containing SSPs, SignalP 5.0, based on deep neural networks, is commonly utilized because it has a user-friendly interface and good performance across plant species ${ }^{71}$. However, since an NSS is common in several types of membrane proteins, membrane spanning proteins with both predicted signal peptide and at least one transmembrane region should be excluded ${ }^{72}$. MEMSAT-SVM ${ }^{73}$ can be used for transmembrane helix topology prediction, and SPOCTOPUS $^{74}$ is designed for predicting both signal peptide and transmembrane topology. Because the existence of certain numbers of NSS-containing proteins follow UPS routes, SecretomeP has been constructed and is a ML algorithm to predict unconventionally secreted proteins $^{75}$. In addition, the number of Cys residues and their arrangement have been used to predict Cys-rich SSPs without signal peptide ${ }^{76}$. In some studies, an additional criterion, such as the lack of endoplasmic reticulum-retention motif, is taken into consideration for secretion prediction. Several authors recommend that small proteins containing C-terminal KDEL or HDEL motifs should be excluded as non-SSPs ${ }^{76,77}$. Protein secretion mediated by conventional (e.g., $\mathrm{CLE}^{78}$ ) or unconventional (e.g., $\mathrm{PME}^{79}$ ) mechanisms can be evaluated using various tools for predicting multiple protein subcellular localizations, such as LocTree3 (refs. ${ }^{80,81}$ ), CELLO $^{82}$, YLoc ${ }^{83}$, DeepLoc ${ }^{84}$, and TargetP ${ }^{85}$. Also, MLbased methods have been developed recently for predicting both conventional and unconventional secretion, e.g., ApoplastP ${ }^{86}$, BUSCA ${ }^{87}$, and Plant-mSubP ${ }^{88}$. A pipeline integrating the best methods for computational prediction of SSPs is proposed in "Integrative approaches for discovery of SSPs". 


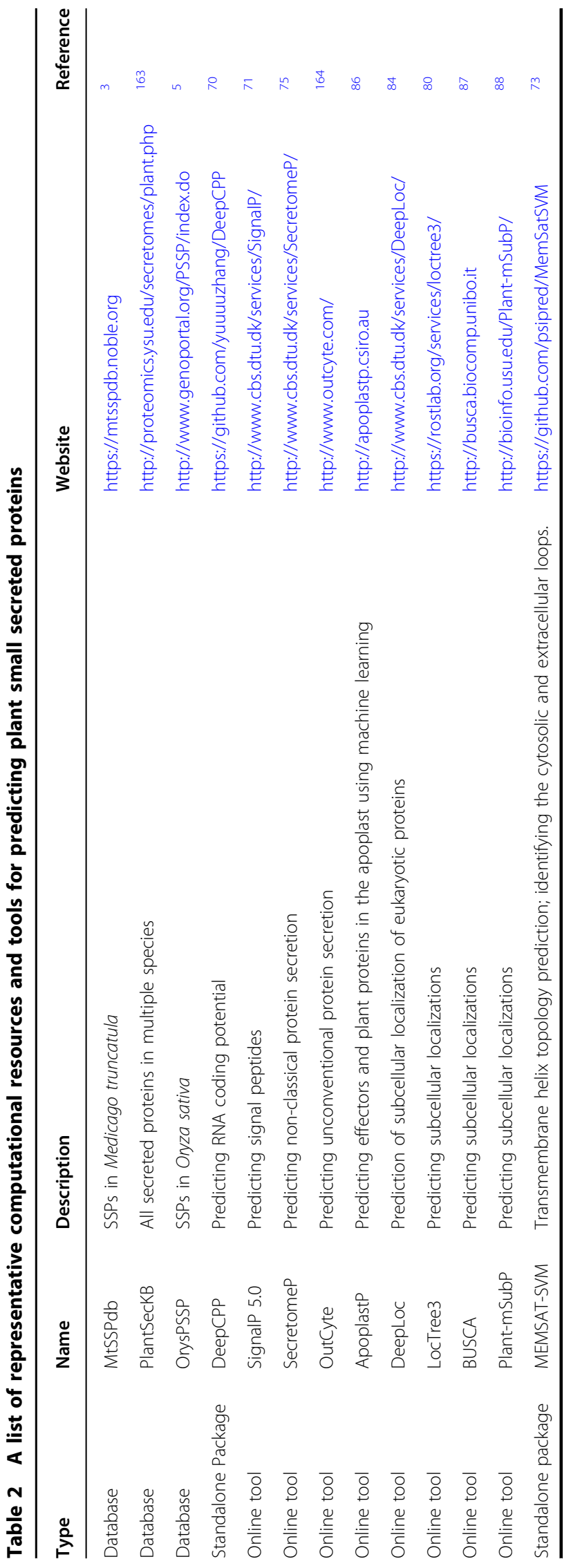

\section{Experimental approaches for discovery of SSPs}

The putative SSPs predicted using computational approaches described in "Computational approaches for discovery of SSPs" need to be verified using experimental approaches to provide protein-level evidence. To address this issue, protein mass spectrometry (MS) data can be used to determine (1) whether the predicted SSPs are truly expressed proteins in extracellular localization and (2) whether the predicted SSP sequences are full length or partial fragments of longer protein sequences. For instance, a novel 15 aa secreted peptide named CEP1 encoded by AT1G47485 was effectively identified in $A$. thaliana by liquid chromatography-mass spectrometry (LC-MS) analysis ${ }^{89}$. The feasibility of this system was tested initially by detecting a known small secreted peptide CLE44 in the medium using transgenic A. thaliana overexpressing the CLE44 gene. Computational prediction of SSP secretion can also be verified through MS analysis of extracellular proteins. For example, protein MS has been successfully used to identify plant immune response proteins that are secreted into apoplastic space in $A$. thaliana leaves ${ }^{90}$. Proteomic analyses of secretomes have identified secreted proteins in O. sativa ${ }^{91}$, Hippophae rhamnoides $^{92}$, S. bicolor ${ }^{54}$, Solanum chacoense ${ }^{93}$, and S. lycopersicum ${ }^{94}$. Such global analyses of plant secretomes could facilitate the discovery of SSPs. However, proteins containing IDRs of sufficient length tend to be more susceptible to degradation, resulting in lower protein abundance ${ }^{26}$. This may cause a problem for studying plant SSPs that contain a large portion of IDRs using proteomics approaches because MS has lower sensitivity than transcriptome sequencing. To increase the sensitivity of detecting SSPs in plants, it is necessary to enrich for IDRs containing proteins and low molecular weight proteins in protein extract using gel filters ${ }^{95}$ or ultrafiltration devices ${ }^{96,97}$.

Besides plant secretome proteomics, molecular approaches can be used to test SSP secretion. For example, the CDS of SSPs can be fused with reporter genes, such as green fluorescent protein $^{98}$, and the gene fusion constructs can be tested for secretion of reportertagged SSPs using agroinfiltration-based transient gene expression $^{99}$ or stable transformation in plants. The secretion of SSPs has been tested using the yeast expression system as well ${ }^{2}$.

\section{Integrative approaches for discovery of SSPs}

From an amalgamation perspective, multiple tools can be assimilated to predict SSPs. Here we propose such a pipeline for SSP discovery by integrating the methods discussed in Sections "Computational approaches for discovery of SSPs" and "Experimental approaches for discovery of SSPs" (as illustrated in Fig. 5). Briefly, sORFs encoding small proteins are predicted from genomic 


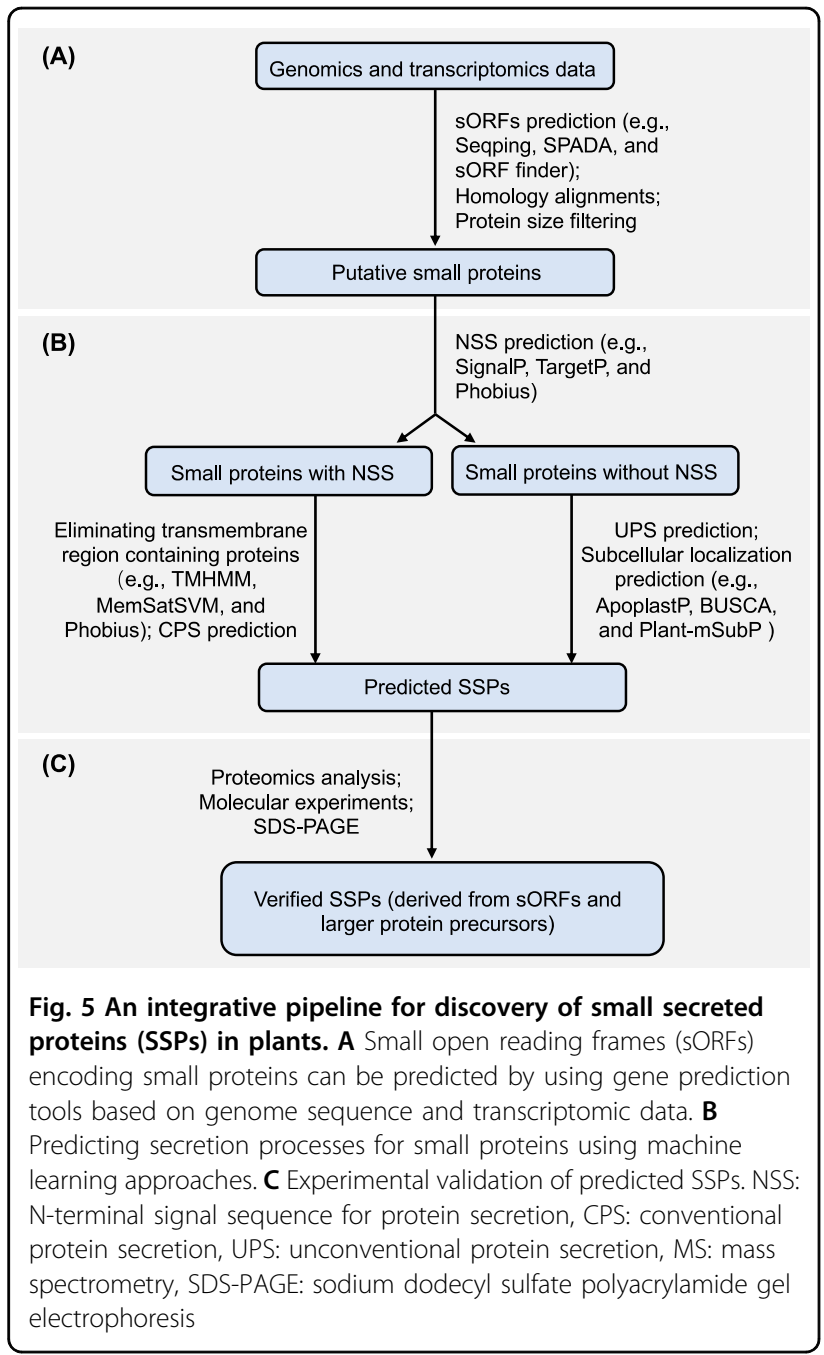

sequences using gene prediction pipeline such as Seqping $^{100}$ based on self-training HMM models and transcriptomic data. Next, NSS-containing small proteins that are transported via CSP pathways are predicted with MLbased tools, such as SignalP 5.0. At this stage small proteins containing transmembrane regions, which are unlikely to be secreted, should be identified and eliminated from downstream analysis. Given that some NSScontaining proteins follow USP pathways, additional ML-based software, such as SecretomeP, may be applied simultaneously. In addition, the secretion ability of proteins without an NSS are inferred by subcellular localization prediction tools (Table 2), which are helpful for predicting secreted proteins contaning an NSS as well. Putative SSPs predicted by computational tools are then validated with MS-based and/or molecular experiments, particularly for their secretion ability, before further functional characterization. Proteomics data are then used to confirm the protein expression of putative sORFs to discover small proteins that are derived from larger protein precursors and/or to localize protein accumulation outside cells.

\section{Strategies for elucidating the function of plant SSPs}

Examination of secretion and transport pathways

Given that apoplastic localization of SSPs can be vital for their function, functional characterization of SSPs often requires refining the knowledge of their trafficking, transport, and secretion routes both within plants and between plants and their microbial partners. Perhaps the most direct method for investigating SSP movement is to visualize SSPs under a fluorescence or electron microscope after tagging them with a fluorescent protein or other label, as demonstrated by Wang et al. ${ }^{101}$ when investigating EXPO-mediated transportation of the A. thaliana Exo70 paralog-Exo70E2, and by Chen et al. ${ }^{102}$ when studying the movement of the transcription factor HY5 from shoot to root in $A$. thaliana. One requirement for this approach is that the fusion of the SSPs and the fluorescent markers must not alter the mobility, secretion, or the function of the $\mathrm{SSPs}^{23,103}$ or interfere with the folding and fluorescence intensity of the markers.

Small-molecule reagents have been used to dissect protein trafficking routes. A widely used example is the fungal toxin brefeldin A (BFA). Given that BFA can disrupt the retrograde traffic from the Golgi to the ER, it serves as a powerful tool for distinguishing Golgidependent and -independent protein trafficking ${ }^{104,105}$. Another example is concanamycin A (ConcA)-an inhibitor of vacuolar-type ATPase (V-ATPase), which blocks post-Golgi trafficking and has been used in examining the transportation pathway of VHA-a3 (refs. ${ }^{106,107}$ ). Additionally, small molecules that can interact with traffickingrelated organelles or vesicles have been used to screen for their potential application in elucidating protein secretion pathways ${ }^{108}$. The power of these trafficking inhibitors, however, becomes limited when it comes to examining the movement of SSPs between plants and microbes. An alternative approach could be based on fluorescently tagged SSP, which was discussed above and appears to be more useful for examining the cross-kingdom movement of plant SSPs.

In addition, a learn-by-design approach based on rewriting the transport pathway can be informative for evaluating if secretion is required for SSP function. Targeted redirection has been achieved by fusing SSPs to alternative sorting signals. For example, Rojo et al. ${ }^{109}$ fused different vacuolar sorting signals to the $\mathrm{C}$ terminus of CLV3 and redirected the destination of CLV3 from apoplast to the vacuole. The authors concluded that apoplastic localization is essential for CLV3 to activate the CLV signaling pathway in A. thaliana. 


\section{Uncovering phenotypic traits conferred by SSP-encoding genes}

Reverse genetics techniques, by imparting loss- or gainof-function mutations via ectopic expression, virusinduced gene silencing, and RNA interference $(\mathrm{RNAi})^{110,111}$, are among the most powerful tools to reveal phenotypes associated with genes of interest. These techniques work equally well for studying the function of SSP-encoding genes. For example, CLV3-the meristem development regulator, when constitutively overexpressed in transgenic $A$. thaliana ${ }^{112}$ demonstrated the correlation between the level of CLV3 protein and the accumulation of the meristem cells. In addition, $A$. thaliana in which the expression of CLV3 was suppressed by RNAi was created by Chuang and Meyerowitz ${ }^{113}$ for studying the associated phenotypic changes in floral development. Similarly, RNAi-induced suppression of the PtCLV3 ortholog PttCLE47 were employed by Kucukoglu et al. ${ }^{114}$ to investigate its role in cambial development and secondary xylem formation in hybrid aspen (Populus tremula $\times$ P. tremuloides).

Besides traditional techniques, the recent revolution in gene editing tools, particularly the invention of the CRISPR/Cas and related technologies, provides new opportunities for efficient gene knockout, gene knockin, gene activation, and gene suppression in plants ${ }^{115-118}$. Its development is based on an immune system naturally found in bacteria and archaea, the CRISPR/Cas9 system has been widely used for creating gene knockouts by creating double-strand breaks, which are then repaired by error-prone the non-homologous end joining in plants and therefore often lead to indel mutations in the target gene. The efficacy of CRISPR/Cas9-mediated gene knockout has been demonstrated in a number of herbaceous and woody plant species ${ }^{119-122}$. In the last few years, the adaptation of CRISPR into a recruiting platform and the discover of Cas9 variants have made CRISPR/Cas a more versatile tool. For example, transcriptional activation and suppression of single and multiple genes can now be conferred by the CRISPR/deactivated Cas9 (dCas9)-based transcriptional regulation system ${ }^{123,124}$. All of these tools can be used in tuning the expression of SSPs for revealing their targets and examining their biological impacts.

\section{Identification of receptors and partners involved in SSP signal transduction pathways}

As discussed above (see "Biological roles of known plant SSPs"), many plant SSPs act as signaling molecules and have the ability to affect the expression of other genes. Therefore, identifying the receptors and other downstream targets of an SSP of interest is the ultimate step towards deciphering SSPs' biological function. A number of early studies, particularly those done in A. thaliana, have been relying on creating targeted mutants or performing mutational screen to achieve this goal. Taking receptors of CLV3 in A. thaliana for instance: CLV1, which is a leucine-rich repeat receptor-like kinase, was verified via phenotypic analysis of single or double mutants $^{125}$. Meanwhile, CORYNE (CRN) which is a membrane-associated protein kinase, and TOADSTOOL2 (TOAD2) which is a receptor-like kinase, were identified by screening the population created with ethyl methanesulfonate mutagenesis ${ }^{126,127}$.

Besides mutational screens, PPI data can provide valuable evidence in identifying novel partners that interact with SSPs during signal transduction. Several in vitro and in vivo PPI detection approaches, such as affinity purification (AP), tandem affinity purification, and yeast twohybrid ( $\mathrm{Y} 2 \mathrm{H})$, have been commonly used ${ }^{128}$. In particular, the capability of $\mathrm{Y} 2 \mathrm{H}$-based approaches has been extended from one-by-one clonal identification to proteomewide mapping of PPIs, with the recent development of matrix-based $\mathrm{Y} 2 \mathrm{H}$ methods coupled with next-generation sequencing (NGS) technology ${ }^{129}$. Compared with mutational screen, Y2H-NGS approaches make it possible to identify novel interaction partners of SSPs even within an organism whose genome has not been fully annotated yet.

\section{Discovery-based extraction, screening, and identification of SSPs}

High-throughput analytical approaches that couple selective enrichment, fractionation/isolation, and phenotype screening followed by MS-based identification provide an established framework to screen plant tissues for biologically relevant SSPs ${ }^{45,89,130-132}$ (Fig. 6). This classical approach for the discovery of novel natural products starts with an enrichment strategy to selectively isolate molecules of interest from highly complex crude extracts. For SSPs, common cellular extraction techniques use size exclusion ultrafiltration strategies, such as molecular weight cut-off spin column filters, to selectively enrich for low molecular weight protein fractions ${ }^{96,97}$. Other techniques include gel-based separations ${ }^{49,95,133}$, solvent extractions $^{89,134}$, and size exclusion chromatography ${ }^{134,135}$. Following these enrichment strategies, SSPs can be further fractionated based on physicochemical properties (e.g., polarity, hydrophobicity, stability, solubility) using liquid chromatography ${ }^{136-138}$.

Either as crude extract mixtures, enrichments, or isolated fractions, SSPs can be evaluated for their bioactivity against cell-based or cell-free biosystems. Cell-based screening can be used to assess simple effects on cell viability, morphology, and proliferation, or to elucidate the mechanism of action. Common phenotypes profiled in cell-based systems are growth promotion/restriction or antimicrobial activity ${ }^{139-142}$. Alternatively, cell-free screening has been employed to evaluate the effect of 


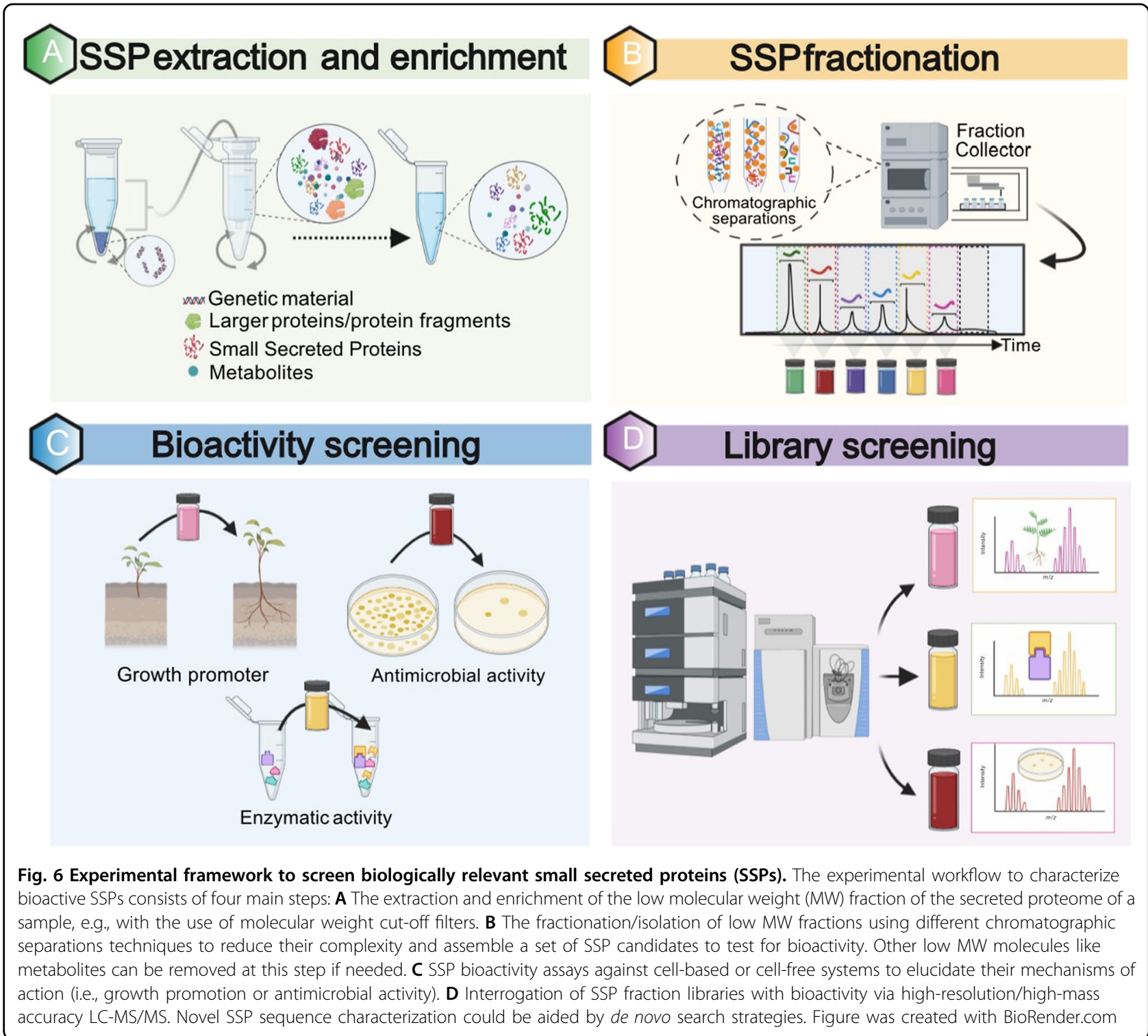

SSPs to better describe the thermodynamic, kinetic, or structural basis for molecular interactions with other cellular constituents ${ }^{143}$. Cell-free screening can be employed to identify SSPs with the abilities to scavenge free radicals, chelate metals, or bind to certain macromolecular targets that regulate various biological processes such as epigenetic processes and cell proliferation $^{144,145}$.

Following the detection of fractions with relevant bioactivity, molecule libraries can be further interrogated via high-throughput LC-MS/MS to sequence unknown SSPs. Some of the current challenges in accurate and sensitive identification of SSPs with MS include lack of SSP representation in protein databases, inadequate understanding of SSP maturation mechanisms, and partial knowledge of their PTM. Thus, the characterization of
SSPs by LC-MS/MS can benefit from the use of de novo search strategies ${ }^{146}$. De novo sequencing algorithms derive peptide sequences using only fragment ion information from the tandem mass spectra, are generally optimized to run without the restriction of cleavage enzymes (i.e., trypsin) and work in an unbiased manner as they do not necessarily require any input based on prior knowledge of the sample ${ }^{147}$.

\section{Conclusion and perspectives}

In the past several years, there has been increasing evidence that SSPs play important roles during plant growth, development and response to biotic and abiotic stresses, and consequently a growing appreciation of the biological significance of plant SSPs. A sheer number of SSPs have been predicted in diverse lineages of organisms, 
and the intercellular or inter-organismal movement of SSPs infers that SSPs are likely a significant and common mode of signaling among organisms. It is now known that SSPs are synthesized and secreted via diverse pathways in plants. Currently, however, the number of characterized SSPs in plants is low. The majority of SSPs encoded in plant genomes are overlooked and remain unannotated. Roadblocks that prevent progress in the study of SSPs include (1) a lack of reliable methods for isolating SSPs for experimental characterization, (2) a lack of capabilities for real-time monitoring the intercellular or inter-organismal movement of SSPs, (3) a lack of structural data for SSPs, and (4) a lack of computational tools for predicting nonconventional secretion of SSPs.

Recent advances in high-throughput molecular screening approaches and bioinformatics offer exciting opportunities for the discovery and characterization of SSPs. For example, the rapid accumulation of omics data, including genomics, transcriptomics, and proteomics, provide rich databases for discovering plant SSPs, including those derived from larger protein precursors and directly encoded by sORFs. Meanwhile, advanced ML tools have evolved to predict the secretion pathways, including both CPS and UPS that SSPs follow. Such computational prediction on secretion can be verified experimentally, for example, via bioimaging of fluorescent reporter-tagged protein candidates. In addition, advanced plant biotechnologies, particularly, CRISPR/Cas-based genomeediting systems and transcriptional regulation systems (i.e., CRISPRa and CRISPRi) allow for efficient gene knockout, activation, and suppression, and therefore analysis of the biological roles of SSPs, and identification of their partners by combining with PPI and NGS data. The discovery and functional role of SSPs in plant growth and development will continue to expand in the near future.

\section{Acknowledgements}

The writing of this manuscript was supported by the Laboratory Directed Research and Development program of Oak Ridge National Laboratory, and the U.S. DOE BER Genomic Science Program, as part of the Secure Ecosystem Engineering and Design and the Plant-Microbe Interfaces Scientific Focus Areas. X.-L.H. received financial support from the China Scholarship Council.

\section{Author details \\ ${ }^{1}$ Department of Plant Sciences, University of Tennessee, Knoxville, TN, USA. Biosciences Division, Oak Ridge National Laboratory, Oak Ridge, TN, USA. ${ }^{3}$ State Key Laboratory of Subtropical Silviculture, School of Forestry and Biotechnology, Zhejiang A\&F University, Hangzhou, Zhejiang, China. ${ }^{4}$ The Center for Bioenergy Innovation, Oak Ridge National Laboratory, Oak Ridge, TN, USA. ${ }^{5}$ Department of Genome Science and Technology, University of Tennessee, Knoxville, TN, USA. ${ }^{6}$ College of Horticulture, Nanjing Agricultural University, Nanjing, Jiangsu, China}

\section{Author contributions}

X.Y. and X.-L.H. conceived the idea. X.-L.H., H.L., and P.E.A. wrote the paper with contributions and input from all authors. All authors reviewed, edited, and accepted the final version of the manuscript.

\section{Conflict of interest}

This manuscript has been authored by UT-Battelle, LLC under Contract No. DEAC05-00OR22725 with the U.S. Department of Energy. The United States Government retains and the publisher, by accepting the article for publication, acknowledges that the United States Government retains a non-exclusive, paid-up, irrevocable, worldwide license to publish or reproduce the published form of this manuscript, or allow others to do so, for United States Government purposes. The Department of Energy will provide public access to these results of federally sponsored research in accordance with the DOE Public Access Plan (http://energy.gov/downloads/doe-public-access-plan). The authors declare no competing interests.

Received: 31 March 2021 Accepted: 26 April 2021

Published online: 01 June 2021

References

1. Lease, K. A. \& Walker, J. C. The Arabidopsis unannotated secreted peptide database, a resource for plant peptidomics. Plant Physiol. 142, 831-838 (2006).

2. Plett, J. M. et al. Populus trichocarpa encodes small, effector-like secreted proteins that are highly induced during mutualistic symbiosis. Sci. Rep. 7, 382 (2017).

3. Boschiero, C. et al. MtSSPdb: the Medicago truncatula small secreted peptide database. Plant Physiol. 183, 399-413 (2020).

4. Chae, K. \& Lord, E. M. Pollen tube growth and guidance: roles of small, secreted proteins. Ann. Bot. 108, 627-636 (2011).

5. Pan, B. et al. OrysPSSP: a comparative platform for small secreted proteins from rice and other plants. Nucleic Acids Res. 41, D1192-D1198 (2012).

6. Sterck, L., Rombauts, S., Vandepoele, K., Rouzé, P., \& Van de Peer, Y. How many genes are there in plants (... and why are they there)?. Curr. Opin. Plant Biol. 10, 199-203 (2007).

7. Boschiero, $\mathrm{C}$. et al. Identification and functional investigation of genomeencoded, small, secreted peptides in plants. Curr. Protoc. Plant Biol. 4, e20098 (2019).

8. Nguyen, T. T., Lee, H.-H., Park, J., Park, I. \& Seo, Y.-S. Computational identification and comparative analysis of secreted and transmembrane proteins in six Burkholderia species. Plant Pathol. J. 33, 148-162 (2017).

9. Krause, C., Richter, S., Knöll, C. \& Jürgens, G. Plant secretome-from cellular process to biological activity. Biochim. Biophys. Acta 1834, 2429-2441 (2013).

10. Yang, X. et al. Discovery and annotation of small proteins using genomics, proteomics, and computational approaches. Genome Res. 21, 634-641 (2011).

11. Tavormina, P., De Coninck, B., Nikonorova, N., De Smet, I. \& Cammue, B. P. A. The plant peptidome: an expanding repertoire of structural features and biological functions. Plant Cell 27, 2095-2118 (2015).

12. Hellens, R. P., Brown, C. M., Chisnall, M. A. W., Waterhouse, P. M. \& Macknight, R. C. The emerging world of small ORFs. Trends Plant Sci. 21, 317-328 (2016).

13. Chen, Y. L., Fan, K. T., Hung, S. C. \& Chen, Y. R. The role of peptides cleaved from protein precursors in eliciting plant stress reactions. N. Phytol. 225, 2267-2282 (2020).

14. Murphy, E., Smith, S. \& De Smet, I. Small signaling peptides in Arabidopsis development: how cells communicate over a short distance. Plant Cell $\mathbf{2 4}$ 3198-3217 (2012).

15. Tabata, R. \& Sawa, S. Maturation processes and structures of small secreted peptides in plants. Front. Plant Sci. 5, 311 (2014).

16. Andrews, S. J. \& Rothnagel, J. A. Emerging evidence for functional peptides encoded by short open reading frames. Nat. Rev. Genet. 15, 193-204 (2014).

17. Hsu, P. Y. \& Benfey, P. N. Small but mighty: functional peptides encoded by small ORFs in plants. Proteomics 18, 1700038 (2018).

18. Rahmani, F. et al. Sucrose control of translation mediated by an upstream open reading frame-encoded peptide. Plant Physiol. 150, 1356-1367 (2009).

19. Lauressergues, D. et al. Primary transcripts of microRNAs encode regulatory peptides. Nature 520, 90-93 (2015).

20. Röhrig, H., Schmidt, J., Miklashevichs, E., Schell, J. \& John, M. Soybean ENOD40 encodes two peptides that bind to sucrose synthase. Proc. Natl Acad. Sci. 99, 1915-1920 (2002).

21. Ding, Y., Robinson, D. G. \& Jiang, L. Unconventional protein secretion (UPS) pathways in plants. Curr. Opin. Cell Biol. 29, 107-115 (2014). 
22. Goring, D. R. \& Di Sansebastiano, G. P. Protein and membrane trafficking routes in plants: conventional or unconventional? J. Exp. Bot. 69, 1-5 (2018)

23. Wang, X., Chung, K. P., Lin, W. \& Jiang, L. Protein secretion in plants: conventional and unconventional pathways and new techniques. J. Exp. Bot. 69 21-37 (2018)

24. Zhang, L., Xing, J. \& Lin, J. At the intersection of exocytosis and endocytosis in plants. N. Phytol. 224, 1479-1489 (2019).

25. Ghorbani, S. et al. Expanding the repertoire of secretory peptides controlling root development with comparative genome analysis and functional assays. J. Exp. Bot. 66, 5257-5269 (2015).

26. van der Lee, R. et al. Classification of intrinsically disordered regions and proteins. Chem. Rev. 114, 6589-6631 (2014).

27. Shinohara, H. \& Matsubayashi, Y. Chemical synthesis of Arabidopsis CLV3 glycopeptide reveals the impact of hydroxyproline arabinosylation on peptide conformation and activity. Plant Cell Physiol. 54, 369-374 (2013).

28. Bobay, B. G. et al. Solution NMR studies of the plant peptide hormone CEP inform function. FEBS Lett. 587, 3979-3985 (2013).

29. Moroder, L., Musiol, H. J., Götz, M. \& Renner, C. Synthesis of single- and multiple-stranded cystine-rich peptides. Biopolymers 80, 85-97 (2005).

30. Mishima, M. et al. Structure of the male determinant factor for Brassica selfincompatibility. J. Biol. Chem. 278, 36389-36395 (2003).

31. Ohki, S., Takeuchi, M. \& Mori, M. The NMR structure of stomagen reveals the basis of stomatal density regulation by plant peptide hormones. Nat. Commun. 2, 1-7 (2011).

32. Fukuda, H. \& Ohashi-Ito, K. Vascular tissue development in plants. Curr. Top. Dev. Biol. 131, 141-160 (2019).

33. Meng, L. \& Feldman, L. J. CLE14/CLE20 peptides may interact with CLAVATA2/CORYNE receptor-like kinases to irreversibly inhibit cell division in the root meristem of Arabidopsis. Planta 232, 1061-1074 (2010)

34. De Smet, I. et al. Receptor-like kinase ACR4 restricts formative cell divisions in the Arabidopsis root. Science 322, 594-597 (2008).

35. Whitford, R., Fernandez, A., De Groodt, R., Ortega, E. \& Hilson, P. Plant CLE peptides from two distinct functional classes synergistically induce division of vascular cells. Proc. Natl Acad. Sci. 105, 18625-18630 (2008).

36. Han, S. et al. Identification and comprehensive analysis of the CLV3/ESRrelated (CLE) gene family in Brassica napus L. Plant Biol. 22, 709-721 (2020).

37. Fukuda, H. \& Hardtke, C. S. Peptide signaling pathways in vascular differentiation. Plant Physiol. 182, 1636 (2020).

38. Etchells, J. P. \& Turner, S. R. The PXY-CLE41 receptor ligand pair defines a multifunctional pathway that controls the rate and orientation of vascular cell division. Development 137, 767-774 (2010)

39. Zhu, Y. et al. A xylem-produced peptide PtrCLE20 inhibits vascular cambium activity in Populus. Plant Biotechnol. J. 18, 195-206 (2020).

40. Fletcher, J. C., Brand, U., Running, M. P., Simon, R. \& Meyerowitz, E. M. Signaling of cell fate decisions by CLAVATA3 in Arabidopsis shoot meristems. Science 283, 1911-1914 (1999).

41. Covey, P. A. et al. A pollen-specific RALF from tomato that regulates pollen tube elongation. Plant Physiol. 153, 703-715 (2010).

42. Chagas, F. O., Pessotti, R. C., Caraballo-Rodriguez, A. M. \& Pupo, M. T. Chemical signaling involved in plant-microbe interactions. Chem. Soc. Rev. 47, 1652-1704 (2018).

43. Segonzac, C. \& Monaghan, J. Modulation of plant innate immune signaling by small peptides. Curr. Opin. Plant Biol. 51, 22-28 (2019).

44. Wang, Y. H. \& Irving, H. R. Developing a model of plant hormone interactions. Plant Signal. Behav. 6, 494-500 (2011).

45. Pearce, G., Strydom, D., Johnson, S. \& Ryan, C. A. A polypeptide from tomato leaves induces wound-inducible proteinase inhibitor proteins. Science $\mathbf{2 5 3}$ 895-897 (1991).

46. Constabel, C. P., Yip, L. \& Ryan, C. A. Prosystemin from potato, black nightshade, and bell pepper: primary structure and biological activity of predicted systemin polypeptides. Plant Mol. Biol. 36, 55-62 (1998)

47. Wang, L. et al. The systemin receptor SYR1 enhances resistance of tomato against herbivorous insects. Nat. Plants 4, 152-156 (2018).

48. Kandoth, P. K. et al. Tomato MAPKs LeMPK1, LeMPK2, and LeMPK3 function in the systemin-mediated defense response against herbivorous insects. Proc Natl Acad. Sci. USA 104, 12205-12210 (2007)

49. Wang, P. et al. Identification of endogenous small peptides involved in rice immunity through transcriptomics- and proteomics-based screening. Plant Biotechnol. J. 18, 415-428 (2020).

50. Zhou, B. et al. Wheat encodes small, secreted proteins that contribute to resistance to Septoria tritici blotch. Front. Genet. 11, 469 (2020).
51. Ziemann, S. et al. An apoplastic peptide activates salicylic acid signalling in maize. Nat. Plants 4, 172-180 (2018).

52. Takahashi, F. et al. A small peptide modulates stomatal control via abscisic acid in long-distance signalling. Nature 556, 235-238 (2018)

53. Atkinson, N. J., Lilley, C. J. \& Urwin, P. E. Identification of genes involved in the response of Arabidopsis to simultaneous biotic and abiotic stresses. Plant Physiol. 162, 2028-2041 (2013).

54. Ngcala, M. G., Goche, T., Brown, A. P., Chivasa, S. \& Ngara, R. Heat stress triggers differential protein accumulation in the extracellular matrix of sorghum cell suspension cultures. Proteomes 8, 29 (2020).

55. Nakaminami, K. et al. AtPep3 is a hormone-like peptide that plays a role in the salinity stress tolerance of plants. Proc. Natl Acad. Sci. USA 115, 5810-5815 (2018).

56. Trivedi, P., Leach, J. E., Tringe, S. G., Sa, T. \& Singh, B. K. Plant-microbiome interactions: from community assembly to plant health. Nat. Rev. Microbiol. 18, 607-621 (2020).

57. Stergiopoulos, I. \& Wit, P. J. G. Md Fungal effector proteins. Annu. Rev. Phytopathol. 47, 233-263 (2009).

58. Kohler, A. et al. Convergent losses of decay mechanisms and rapid turnover of symbiosis genes in mycorrhizal mutualists. Nat. Genet. 47, 410-415 (2015)

59. Péret, B., Larrieu, A. \& Bennett, M. J. Lateral root emergence: a difficult birth. J. Exp. Bot. 60, 3637-3643 (2009).

60. Imin, N., Mohd-Radzman, N. A., Ogilvie, H. A. \& Djordjevic, M. A. The peptideencoding CEP1 gene modulates lateral root and nodule numbers in Medicago truncatula. J. Exp. Bot. 64, 5395-5409 (2013).

61. Gonzalez-Rizzo, S, Crespi, M. \& Frugier, F. The Medicago truncatula CRE1 cytokinin receptor regulates lateral root development and early symbiotic interaction with Sinorhizobium meliloti. Plant Cell 18, 2680-2693 (2006).

62. Whitford, R. et al. GOLVEN secretory peptides regulate auxin carrier turnover during plant gravitropic responses. Dev. Cell 22, 678-685 (2012).

63. Laffont, $C$. et al. The NIN transcription factor coordinates CEP and CLE signaling peptides that regulate nodulation antagonistically. Nat. Commun. 11 1-13 (2020).

64. Handa, Y. et al. RNA-seq transcriptional profiling of an arbuscular mycorrhiza provides insights into regulated and coordinated gene expression in Lotus japonicus and Rhizophagus irregularis. Plant Cell Physiol. 56, 1490-1511 (2015).

65. Martinez, T. F. et al. Accurate annotation of human protein-coding small open reading frames. Nat. Chem. Biol. 16, 458-468 (2020).

66. Hanada, K. et al. sORF finder: a program package to identify small open reading frames with high coding potential. Bioinformatics 26, 399-400 (2010)

67. Zhou, P. et al. Detecting small plant peptides using SPADA (small peptide alignment discovery application. BMC Bioinformatics 14, 335 (2013).

68. Liu, D., Mewalal, R., Hu, R., Tuskan, G. A. \& Yang, X. New technologies accelerate the exploration of non-coding RNAs in horticultural plants. Hortic. Res. 4, 17031 (2017).

69. Mewalal, R. et al. Identification of populus small RNAs responsive to mutualistic interactions with mycorrhizal fungi, Laccaria bicolor and Rhizophagus irregularis. Front. Microbiol. 10, 515 (2019).

70. Zhang, Y., Jia, C., Fullwood, M. J. \& Kwoh, C. K. DeepCPP: a deep neural network based on nucleotide bias information and minimum distribution similarity feature selection for RNA coding potential prediction. Brief. Bioinformatics 22, 2073-2084 (2020).

71. Almagro Armenteros, J. J. et al. SignalP 5.0 improves signal peptide predictions using deep neural networks. Nat. Biotechnol. 37, 420-423 (2019).

72. Uhlén, M. et al. Tissue-based map of the human proteome. Science $\mathbf{3 4 7}$ 1260419 (2015).

73. Nugent, T. \& Jones, D. T. Detecting pore-lining regions in transmembrane protein sequences. BMC Bioinformatics 13, 1-9 (2012).

74. Viklund, H., Bernsel, A., Skwark, M. \& Elofsson, A. SPOCTOPUS: a combined predictor of signal peptides and membrane protein topology. Bioinformatics 24, 2928-2929 (2008).

75. Nielsen, H., Petsalaki, E. I., Zhao, L. \& Stühler, K. Predicting eukaryotic protein secretion without signals. Biochim. Biophys. Acta 1867, 140174 (2019).

76. Li, Y. L., Dai, X. R, Yue, X, Gao, X.-O \& Zhang, X. S. Identification of small secreted peptides (SSPS) in maize and expression analysis of partial SSP genes in reproductive tissues. Planta 240, 713-728 (2014).

77. de Bang, T. C. et al. Genome-wide identification of Medicago peptides involved in macronutrient responses and nodulation. Plant Physiol. 175 1669-1689 (2017). 
78. Whitewoods, C. Evolution of CLE peptide signalling. Semin. Cell Dev. Biol. 109, 12-19 (2021).

79. Wang, $\mathrm{H}$. et al. A distinct pathway for polar exocytosis in plant cell wall formation. Plant Physiol. 172, 1003-1018 (2016).

80. Goldberg, T. et al. LocTree3 prediction of localization. Nucleic Acids Res. 42, W350-W355 (2014).

81. Goldberg, T., Hamp, T. \& Rost, B. LocTree2 predicts localization for all domains of life. Bioinformatics 28, i458-i465 (2012).

82. Yu, C. S., Chen, Y. C., Lu, C. H. \& Hwang, J. K. Prediction of protein subcellular localization. Proteins Struct. Funct. Bioinformatics 64, 643-651 (2006).

83. Briesemeister, S., Rahnenführer, J. \& Kohlbacher, O. YLoc-an interpretable web server for predicting subcellular localization. Nucleic Acids Res. 38 W497-W502 (2010)

84. Almagro Armenteros, J. J., Sønderby, C. K., Sønderby, S. K., Nielsen, H. \& Winther, O. DeepLoc: prediction of protein subcellular localization using deep learning. Bioinformatics 33, 3387-3395 (2017).

85. Armenteros, J. J. A. et al. Detecting sequence signals in targeting peptides using deep learning. Life Sci. Alliance 2, e201900429 (2019).

86. Sperschneider, J., Dodds, P. N., Singh, K. B. \& Taylor, J. M. ApoplastP: prediction of effectors and plant proteins in the apoplast using machine learning. $N$. Phytol. 217, 1764-1778 (2018)

87. Savojardo, C., Martelli, P. L., Fariselli, P., Profiti, G. \& Casadio, R. BUSCA: an integrative web server to predict subcellular localization of proteins. Nucleic Acids Res. 46, W459-w466 (2018).

88. Sahu, S. S., Loaiza, C. D. \& Kaundal, R. Plant-mSubP: a computational framework for the prediction of single- and multi-target protein subcellular localization using integrated machine-learning approaches. AoB Plants 12, plz068 (2019).

89. Ohyama, K., Ogawa, M. \& Matsubayashi, Y. Identification of a biologically active, small, secreted peptide in Arabidopsis by in silico gene screening followed by LC-MS-based structure analysis. Plant J. 55, 152-160 (2008).

90. Rutter, B. D. \& Innes, R. W. Extracellular vesicles isolated from the leaf apoplast carry stress-response proteins. Plant Physiol. 173, 728-741 (2017).

91. Shinano, T. et al. Proteomic analysis of secreted proteins from aseptically grown rice. Phytochemistry 72, 312-320 (2011).

92. Gupta, R. \& Deswal, R. Low temperature stress modulated secretome analysis and purification of antifreeze protein from Hippophae rhamnoides, a Himalayan wonder plant. J. Proteome Res. 11, 2684-2696 (2012).

93. Liu, Y., Joly, V., Dorion, S., Rivoal, J. \& Matton, D. P. The plant ovule secretome: a different view toward pollen-pistil interactions. J. Proteome Res. 14 4763-4775 (2015).

94. Briceño, Z. et al. Enhancement of phytosterols, taraxasterol and induction of extracellular pathogenesis-related proteins in cell cultures of Solanum lycopersicum cv Micro-Tom elicited with cyclodextrins and methyl jasmonate. J. Plant Physiol. 169, 1050-1058 (2012)

95. Chen, L. et al. Development of gel-filter method for high enrichment of lowmolecular weight proteins from serum. PLOS ONE 10, e0115862-e0115862 (2015).

96. Greening, D. W. \& Simpson, R. J. A centrifugal ultrafiltration strategy for isolating the low-molecular weight $(\leq 25 \mathrm{~K})$ component of human plasma proteome. J. Proteomics 73, 637-648 (2010).

97. Villalobos Solis, M. I. et al. A viable new strategy for the discovery of peptide proteolytic cleavage products in plant-microbe interactions. Mol. Plant Microbe Interact. 33, 1177-1188 (2020)

98. Zhang, L. et al. The Verticillium-specific protein VdSCP7 localizes to the plant nucleus and modulates immunity to fungal infections. N. Phytol. 215 368-381 (2017).

99. Norkunas, K., Harding, R., Dale, J. \& Dugdale, B. Improving agroinfiltrationbased transient gene expression in Nicotiana benthamiana. Plant Methods 14, 71 (2018).

100. Chan, K.-L. et al. Seqping: gene prediction pipeline for plant genomes using self-training gene models and transcriptomic data. BMC Bioinformatics $\mathbf{1 8}$ 1-7 (2017)

101. Wang, J. et al. EXPO, an exocyst-positive organelle distinct from multivesicular endosomes and autophagosomes, mediates cytosol to cell wall exocytosis in Arabidopsis and tobacco cells. Plant Cell 22, 4009-4030 (2010).

102. Chen, $X$. et al. Shoot-to-root mobile transcription factor HY5 coordinates plant carbon and nitrogen acquisition. Curr. Biol. 26, 640-646 (2016).

103. Burko, Y., Gaillochet, C., Seluzicki, A., Chory, J. \& Busch, W. Local HY5 activity mediates hypocotyl growth and shoot-to-root communication. Plant Commun. 1, 100078 (2020)
104. Pinedo, M. et al. Extracellular sunflower proteins: evidence on non-classical secretion of a jacalin-related lectin. Protein Pept. Lett. 19, 270-276 (2012).

105. Zhang, $\mathrm{H}$. et al. Golgi apparatus-localized synaptotagmin 2 is required for unconventional secretion in Arabidopsis. PLoS ONE 6, e26477 (2011).

106. Scheuring, D. et al. Multivesicular bodies mature from the trans-Golgi network/early endosome in Arabidopsis. Plant Cell 23, 3463-3481 (2011).

107. Viotti, $C$. et al. The endoplasmic reticulum is the main membrane source for biogenesis of the lytic vacuole in Arabidopsis. Plant Cell 25, 3434-3449 (2013).

108. Rodriguez-Furlan, C., Raikhel, N. V. \& Hicks, G. R. Merging roads: chemical tools and cell biology to study unconventional protein secretion. J. Exp. Bot. 69 39-46 (2018).

109. Rojo, E., Sharma, V. K., Kovaleva, V., Raikhel, N. V. \& Fletcher, J. C. CLV3 is localized to the extracellular space, where it activates the Arabidopsis CLAVATA stem cell signaling pathway. Plant Cell 14, 969-977 (2002).

110. Ben-Amar, A., Daldoul, S. M., Reustle, G., Krczal, G. \& Mliki, A. Reverse genetics and high throughput sequencing methodologies for plant functional genomics. Curr. Genomics 17, 460-475 (2016).

111. Gilchrist, E. \& Haughn, G. Reverse genetics techniques: engineering loss and gain of gene function in plants. Brief. Funct. Genomics 9, 103-110 (2010).

112. Brand, U., Fletcher, J. C., Hobe, M., Meyerowitz, E. M. \& Simon, R. Dependence of stem cell fate in Arabidopsis on a feedback loop regulated by CLV3 activity. Science 289, 617-619 (2000)

113. Chuang, C.-F. \& Meyerowitz, E. M. Specific and heritable genetic interference by double-stranded RNA in Arabidopsis thaliana. Proc. Natl Acad. Sci. 97 4985-4990 (2000).

114. Kucukoglu, M. et al. Peptide encoding Populus CLV3/ESR-RELATED 47 (PttCLE47) promotes cambial development and secondary xylem formation in hybrid aspen. N. Phytol. 226, 75-85 (2020)

115. Yang, $X$. et al. Plant biosystems design research roadmap 1.0. BioDesign Res. 2020, 8051764 (2020).

116. Hassan, M. M., Yuan, G., Chen, J.-G., Tuskan, G. A. \& Yang, X. Prime editing technology and its prospects for future applications in plant biology research. BioDesign Res. 2020, 9350905 (2020).

117. Zhang, Y. \& Qi, Y. Diverse systems for efficient sequence insertion and replacement in precise plant genome editing. BioDesign Res. 2020, 8659064 (2020).

118. Liu, D., Hu, R., Palla, K. J., Tuskan, G. A. \& Yang, X. Advances and perspectives on the use of CRISPR/Cas9 systems in plant genomics research. Curr. Opin. Plant Biol. 30, 70-77 (2016).

119. Elorriaga, E., Klocko, A. L., Ma, C. \& Strauss, S. H. Variation in mutation spectra among CRISPR/Cas9 mutagenized poplars. Front. Plant Sci. 9, 594 (2018).

120. Li, J., Li, Y. \& Ma, L. CRISPR/Cas9-based genome editing and its applications for functional genomic analyses in plants. Small Methods 3, 1800473 (2019).

121. Liu, D. et al. CRISPR/Cas9-mediated targeted mutagenesis for functional genomics research of crassulacean acid metabolism plants. J. Exp. Bot. 70 6621-6629 (2019).

122. Xue, L.-J., Alabady, M. S., Mohebbi, M. \& Tsai, C.-J. Exploiting genome variation to improve next-generation sequencing data analysis and genome editing efficiency in Populus tremulax alba 717-1B4. Tree Genet. Genomes 11, 1-8 (2015).

123. Lowder, L. G., Paul, J. W. \& Qi, Y. Plant Gene Regulatory Networks. Methods in Molecular Biology, Vol. 1629 (eds. Kaufmann, K. \& Mueller-Roeber, B.) 167-184 (Humana Press, 2017).

124. Zhang, Y., Malzahn, A. A., Sretenovic, S. \& Qi, Y. The emerging and uncultivated potential of CRISPR technology in plant science. Nat. Plants 5, 778-794 (2019).

125. Clark, S. E., Running, M. P. \& Meyerowitz, E. M. CLAVATA3 is a specific regulator of shoot and floral meristem development affecting the same processes as CLAVATA1. Development 121, 2057-2067 (1995).

126. Kinoshita, A. et al. RPK2 is an essential receptor-like kinase that transmits the CIV3 signal in Arabidopsis. Development 137, 3911-3920 (2010).

127. Müller, R., Bleckmann, A. \& Simon, R. The receptor kinase CORYNE of Arabidopsis transmits the stem cell-limiting signal CLAVATA3 independently of CLAVATA1. Plant Cell 20, 934-946 (2008).

128. Rao, V. S. Srinivas, K, Sujini, G. \& Kumar, G. Protein-protein interaction detection: methods and analysis. Int. J. Proteomics 2014, 147648 (2014).

129. Erffelinck, M.-L. et al. A user-friendly platform for yeast two-hybrid library screening using next generation sequencing. PLOS ONE 13, e0201270 (2018).

130. Demarque, D. P. et al. Mass spectrometry-based metabolomics approach in the isolation of bioactive natural products. Sci. Rep. 10, 1-9 (2020). 
131. Cao, B. et al. Seeing the unseen of the combination of two natural resins, frankincense and myrrh: changes in chemical constituents and pharmacological activities. Molecules 24, 3076 (2019).

132. Pearce, G., Moura, D. S., Stratmann, J. \& Ryan, C. A. Production of multiple plant hormones from a single polyprotein precursor. Nature 411, 817-820 (2001).

133. Cheli, F. \& Baldi, A. Nutrition-based health: cell-based bioassays for food antioxidant activity evaluation. J. Food Sci. 76, R197-R205 (2011).

134. Patel, N. et al. Diverse peptide hormones affecting root growth identified in the Medicago truncatula secreted peptidome. Mol. Cell. Proteomics 17 160-174 (2018).

135. Mohd-Radzman, N. A. et al. Novel MtCEP1 peptides produced in vivo differentially regulate root development in Medicago truncatula. J. Exp. Bot. 66 5289-5300 (2015).

136. Wilson, B. A., Thornburg, C. C., Henrich, C. J., Grkovic, T. \& O'Keefe, B. R. Creating and screening natural product libraries. Nat. Prod. Rep. 37, 893-918 (2020)

137. Kim, Y.-G., Lone, A. M. \& Saghatelian, A. Analysis of the proteolysis of bioactive peptides using a peptidomics approach. Nat. Protoc. 8, 1730 (2013).

138. Alexandersson, E., Ashfaq, A., Resjö, S. \& Andreasson, E. Plant secretome proteomics. Front. Plant Sci. 4, 9 (2013).

139. Ito, Y. et al. Dodeca-CLE peptides as suppressors of plant stem cell differentiation. Science 313, 842-845 (2006)

140. Matsubayashi, Y. \& Sakagami, Y. Phytosulfokine, sulfated peptides that induce the proliferation of single mesophyll cells of Asparagus officinalis L. Proc. Natl Acad. Sci. 93, 7623-7627 (1996).

141. Runyoro, D. K., Matee, M. I., Ngassapa, O. D., Joseph, C. C. \& Mbwambo, Z. H. Screening of Tanzanian medicinal plants for anti-Candida activity. BMC Complement. Altern. Med. 6, 1-10 (2006)

142. Mabona, U., Viljoen, A., Shikanga, E., Marston, A. \& Van Vuuren, S. Antimicrobial activity of southern African medicinal plants with dermatological relevance: from an ethnopharmacological screening approach, to combination studies and the isolation of a bioactive compound. J. Ethnopharmacol. 148, 45-55 (2013).

143. Makarewich, C. A. \& Olson, E. N. Mining for micropeptides. Trends Cell Biol. 27 685-696 (2017).

144. Nwachukwu, I. D. \& Aluko, R. E. Structural and functional properties of food protein-derived antioxidant peptides. J. Food Biochem. 43, e12761 (2019)

145. Ding, $M$. et al. Secretome-based screening in target discovery. SLAS Discov. 25, 535-551 (2020).

146. Cheng, Q. et al. Identifying secreted proteins of Marssonina brunnea by degenerate PCR. Proteomics 10, 2406-2417 (2010).

147. Ma, B. \& Johnson, R. De novo sequencing and homology searching. Mol. Cell. Proteomics 11, 0111-014902 (2012).

148. Kondo, T. et al. A plant peptide encoded by CLV3 identified by in situ MALDITOF MS analysis. Science 313, 845-848 (2006).

149. Hunt, L., Bailey, K. J. \& Gray, J. E. The signalling peptide EPFL9 is a positive regulator of stomatal development. New Phytol 186, 609-614 (2010).

150. Hara, K., Kajita, R., Torii, K. U., Bergmann, D. C. \& Kakimoto, T. The secretory peptide gene EPF1 enforces the stomatal one-cell-spacing rule. Genes Dev. 21, 1720-1725 (2007)

151. Fernandez, A. et al. The GLV6/RGF8/CLEL2 peptide regulates early pericycle divisions during lateral root initiation. J. Exp. Bot. 66, 5245-5256 (2015).
152. Potocka, I., Baldwin, T. C. \& Kurczynska, E. U. Distribution of lipid transfer protein 1 (LTP1) epitopes associated with morphogenic events during somatic embryogenesis of Arabidopsis thaliana. Plant Cell Rep. 31, 2031-2045 (2012)

153. Hou, S. et al. The secreted peptide PIP1 amplifies immunity through receptor-like kinase 7. PLoS Pathog. 10, e1004331 (2014).

154. Huffaker, A., Pearce, G. \& Ryan, C. A. An endogenous peptide signal in Arabidopsis activates components of the innate immune response. Proc. Natl Acad. Sci. 103, 10098-10103 (2006).

155. Ross, A. et al. The Arabidopsis PEPR pathway couples local and systemic plant immunity. EMBO J. 33, 62-75 (2014).

156. Mosher, S. et al. The tyrosine-sulfated peptide receptors PSKR1 and PSY1R modify the immunity of Arabidopsis to biotrophic and necrotrophic pathogens in an antagonistic manner. Plant J. 73, 469-482 (2013).

157. Sharma, A. et al. Comprehensive analysis of plant rapid alkalization factor (RALF) genes. Plant Physiol. Biochem. 106, 82-90 (2016).

158. Matsuzaki, Y., Ogawa-Ohnishi, M., Mori, A. \& Matsubayashi, Y. Secreted peptide signals required for maintenance of root stem cell niche in Arabidopsis. Science 329, 1065-1067 (2010).

159. Santiago, J. et al. Mechanistic insight into a peptide hormone signaling complex mediating floral organ abscission. Elife 5, e15075 (2016).

160. Horváth, B. et al. Loss of the nodule-specific cysteine rich peptide, NCR169, abolishes symbiotic nitrogen fixation in the Medicago truncatula dnf7 mutant. Proc. Natl Acad. Sci. USA 112, 15232-15237 (2015).

161. Weerawanich, K., Webster, G., Ma, J. K., Phoolcharoen, W. \& Sirikantaramas, S Gene expression analysis, subcellular localization, and in planta antimicrobial activity of rice (Oryza sativa L.) defensin 7 and 8. Plant Physiol. Biochem. 124, 160-166 (2018).

162. Chen, Y.-L. et al. Quantitative peptidomics study reveals that a woundinduced peptide from PR-1 regulates immune signaling in tomato. Plant Cell 26, 4135-4148 (2014).

163. Lum, G., Meinken, J., Orr, J., Frazier, S. \& Min, X. J. PlantSecKB: the plant secretome and subcellular proteome knowledgebase. Comput. Mol. Biol. 4, 1-17 (2014)

164. Zhao, L. et al. OutCyte: a novel tool for predicting unconventional protein secretion. Sci. Rep. 9, 19448 (2019).

165. Burley, S. K. et al. RCSB Protein Data Bank: powerful new tools for exploring 3D structures of biological macromolecules for basic and applied research and education in fundamental biology, biomedicine, biotechnology, bioengineering and energy sciences. Nucleic Acids Res. 49, D437-D451 (2020)

166. Berman, H. M. et al. The Protein Data Bank. Nucleic Acids Res. 28, 235-242 (2000).

167. Sehnal, D., Rose, A., Koča, J., Burley, S. \& Velankar, S. Mol* towards a common library and tools for web molecular graphics. Proc. Workshop on Molecular Graphics and Visual Analysis of Molecular Data 29-33 (2018).

168. Goodstein, D. M. et al. Phytozome: a comparative platform for green plant genomics. Nucleic Acids Res. 40, D1178-D1186 (2012).

169. Mészáros, B., Erdős, G. \& Dosztányi, Z. IUPred2A: context-dependent prediction of protein disorder as a function of redox state and protein binding Nucleic Acids Res. 46, W329-W337 (2018).

170. Erdős, G. \& Dosztányi, Z. Analyzing protein disorder with IUPred2A. Curr Protoc. Bioinformatics 70, e99 (2020). 